\title{
Extending the choice architecture toolbox: The Choice Context Mapping
}

Nandor Hajdu ${ }^{\text {ab }}$, Barnabas Szaszi ${ }^{b}$, Balazs Aczel ${ }^{b}$

${ }^{a}$ Doctoral School of Psychology, ELTE Eotvos Lorand University, Budapest, Hungary

${ }^{b}$ Institute of Psychology, ELTE Eotvos Lorand University, Budapest, Hungary

This project was supported by the Hungarian National Research, Development and Innovation Office (NKFIH-11578/2019-DT).

*Author for correspondence: hajdu.nandor93@gmail.com

Address: Institute of Psychology, ELTE Eotvos Lorand University, Izabella u. 46, 1064, Budapest, Hungary 


\begin{abstract}
The role of context in behavioral interventions is indisputable, yet few intervention studies start with a systematic mapping of the influencing contextual factors. This is mostly due to the lack of a methodology that researchers can employ for this aim. Recognizing this current limitation of the field, we developed a procedure, the Choice Context Mapping, to provide researchers a tool to examine the contextual factors of a targeted behavior. We demonstrate the steps of Choice Context Mapping on the behavioural choice situation of stairs vs. elevator use. Potential contextual factors were collected from laypeople as well as experts, and two surveys were created to measure both the behavior and choice, as well as the beliefs of participants. We estimated the effect of contextual factors on the participants' behavior and managed to identify the most influential ones regarding their contribution to the studied choice. Using Choice Context Mapping, we obtained an accurate prediction of whether one chooses the stairs or elevator based on contextual information in $93.26 \%$ of the cases, on previously unseen data. We also ascertained that participants differed in their beliefs about what influences them in this particular choice, and that they can be divided into different groups based on their beliefs. Our results indicate that Choice Context Mapping is a useful procedure for the collection and assessment of contextual factors in a given choice setting which can help the planning of behavioral interventions by considerably lowering the number of possible interventions that are plausibly effective.
\end{abstract}

Keywords: Choice Context Mapping, choice architecture; nudge; behavioral interventions; generalizability 


\section{Introduction}

Imagine you wish to nudge people towards a healthy behavior, such as taking the stairs instead of using the elevator. The literature shows plenty of different nudge techniques in different setups with varying degrees of success. How should you decide which nudge technique to use? We argue that researchers cannot make an informed decision about their intervention technique until influential contextual factors of the studied choice situations are explored. This paper provides procedural steps to detect contextual influences to assist choice architecture interventions.

The headway of nudge interventions, as methods for changing behavior on a large scale, became apparent in the last decade. Nudge theory, as described in Thaler and Sunstein's book (2008), advocates influencing people's behavior via the alteration of choice architecture with relatively inexpensive and non-invasive methods by building on general cognitive processes and biases. While success can be achieved with the help of nudges in many cases (John et al., 2013), sometimes these same methods prove to be ineffective (e.g., Silva \& John, 2017), or their effect is not persistent (Brandon et al., 2017).

One of the potential reasons for this inconsistency of results might be that in the prevalent culture of behavioral intervention research, nudge researchers intend to find all-encompassing effect sizes and do not consider the potential heterogeneity across various contexts (Tipton et al., 2019). Thus, the explanation of what, when, why and to what extent interventions work remains untold. It is suggested that theory advancement and the exploration of moderators should be pursued instead of trial-and-error assessment of ad hoc interventions in a given context (Szaszi et al., 2018).

\section{The focus is on context}

We define context as both the physical attributes of the environment as well as nonphysical factors (e.g., social or psychological circumstances) which might have an effect on decisions. We argue that knowing the context while planning interventions is a necessary, whilst not sufficient, prerequisite of intervention choice. Mapping potential influencing factors while there is no intervention in effect is needed to later model their interactions with the effects of interventions. This way, it is possible to advance the general understanding of how the interventions work. 
Sufficient knowledge of context has key importance. In this sense, mapping contextual information is analogous to an anamnesis in therapy settings; while the therapeutic methods that will be used cannot be decided based solely on the anamnesis, it is still a vital part of the method as it helps in designating further directions.

The role of context in interventions is rarely completely ignored, yet its investigation is seldom systematic. Regarding the context of the planned intervention, two main sources of information indicate which potential contextual information to heed: it is either based on some insight, without empirical data, or on published results.

Insight based contextual information does not necessarily stem from the researchers' own experience and opinion; it can also originate from other experts' opinions - collected either via interviews or just plain conversation about the topic. Valuable information can also be gathered by summoning a demographically diverse focus group and discussing the matter extensively (Puchta \& Potter, 2004). However, the insight approach has its drawbacks: its strength depends on the validity and breadth of these insights, something that is rarely known or assessed.

Empirical contextual information might be acquired from relevant literature: specific interventions, reviews, and meta-analyses. The main limitation of these sources is that the generalizability of the findings is limited to the context of the original studies, and there are only a very few studies exploring the generalizability question (Szaszi et al., 2018). Furthermore, we cannot possibly know the contextual influences without their previous in depth exploration.

\section{Existing frameworks}

There are numerous frameworks and guidelines that can be of assistance when planning interventions. For example, MINDSPACE (Dolan et al., 2010) is intended to provide a more indepth aid, describing 9 robust influences on human behavior: Messenger, Incentives, Norms, Defaults, Salience, Priming, Affect, Commitments and Ego, most of which can be context-related. The guide of Ly, Mažar, Zhao and Soman (2013) accentuates the importance of contextual mapping, and provides a useful set of questions about the properties of the decision, such as incentives and cost, sources of information for the individual making the decision, features of the individuals' mindset and various environmental factors which can help in planning interventions. However, there are no instructions provided on how to answer these questions. EAST (Algate et 
al., n.d.), is a purposefully simple framework to follow, aimed at policy-makers rather than researchers. It is argued that planning and executing a behavioral intervention can be more successful if several attributes of the intervention are set before planning smaller details: it should be Easy, Attractive, Social, and Timely. Another framework, the BASIC approach developed by the iNudgeyou team (Schmidt et al., 2016) offers a guideline for planning interventions with an emphasis on applicability. The first step is Behavioral mapping, which is the collection of data via observations of the target population. While observation is a valuable source of information, it does not necessarily further the understanding of the reasons and motivations behind the behavior. While these frameworks certainly help in designing nudge interventions, and some of them emphasize the importance of contextual information, they do not provide a standardized method to investigate and measure these factors. The exact methods of prior assessment are left for the reader to contrive. We argue that the lack of thorough and comprehensive mapping of moderators and potential contextual influences is one of the main obstacles in developing extensive theoretical frameworks of large-scale behavioral interventions (Szaszi et al., 2018).

\section{Choice Context Mapping}

We developed the Choice Context Mapping, a procedure that aims to assist the researcher to explore the influential contextual factors in a given choice situation. The procedure consists of four steps.

\section{Step 1: Collecting Potential Influencing Factors}

The goal here is to gather information from diverse sources, including the relevant professional literature, the target population, as well as experts about what attributes of the context (aka. factors) might influence the given choice. Such factors can refer to the physical attributes of the environment, the nonphysical factors such as social, cultural, or psychological attributes of the target population as well as the timing of the choice. This step can be achieved through asking experts and laypeople (e.g., by questionnaires, interviews or focus group discussions) about the potential influencing factors of the behavior in question. This exploration can bring details to the surface that are not available from the literature. In order to create a final list of potential factors, the collection needs to be curated by merging all elements referring to the same attributes of the context. 


\section{Step 2: Quantifying the Influence of Factors}

The aim of Step 2 is (a) to measure each contextual factor that potentially influences the choice in question, along with a measurement of the choices themselves, as well as (b) to understand the extent to which each contextual factor contributes to the observed behavior. The data collected can be used to estimate the strength and direction of the relationship between the observed behavior and the gathered contextual factors. When planning interventions, such estimates can be used to predict change in behavior when these contextual factors change. Collecting behavioral data in natural settings has its value as people's beliefs about and observations on choice behavior might not fully overlap.

\section{Step 3: Assessing Beliefs about the Influence of Factors}

The aim is to measure (a) the extent to which people believe that the factors collected in Step 1 influence their behavior in a choice context and also (b) to explore whether people can be grouped based on their beliefs about what influences their elevator/stair choices, and, if possible, the relative sizes of these groups. First, a survey that allows quantitative measurements to be taken must be created in order to assess the beliefs of individuals. Then, it must be verified whether meaningful clusters of the sample can be formulated based on beliefs of the contextual influences. These clusters provide information about which beliefs occur together, as well as the relative ratios of people having the same thinking about the situation. This can be helpful when planning interventions as utilizing cues that the most people are sensitive to might potentially have the largest impact overall.

\section{Comparative analysis}

For those who wish to find out whether people's choice-related beliefs and their corresponding behavior are aligned, we recommend the comparison of the results of Steps 2 and 3. This analysis can indicate how much people are aware of the causes of their choice in a given context, or whether they hold false beliefs about their behavior. It can be informative about how much we can rely on people's insights in understanding the choice context. It might not be possible to compare models defining the relationships between the contextual factors and behavior, and the models describing beliefs about these contextual factors directly. However, the relative order of effect sizes can be contrasted, and 
Following this procedure, relevant contextual factors can be identified and their effects on the target behavior measured.

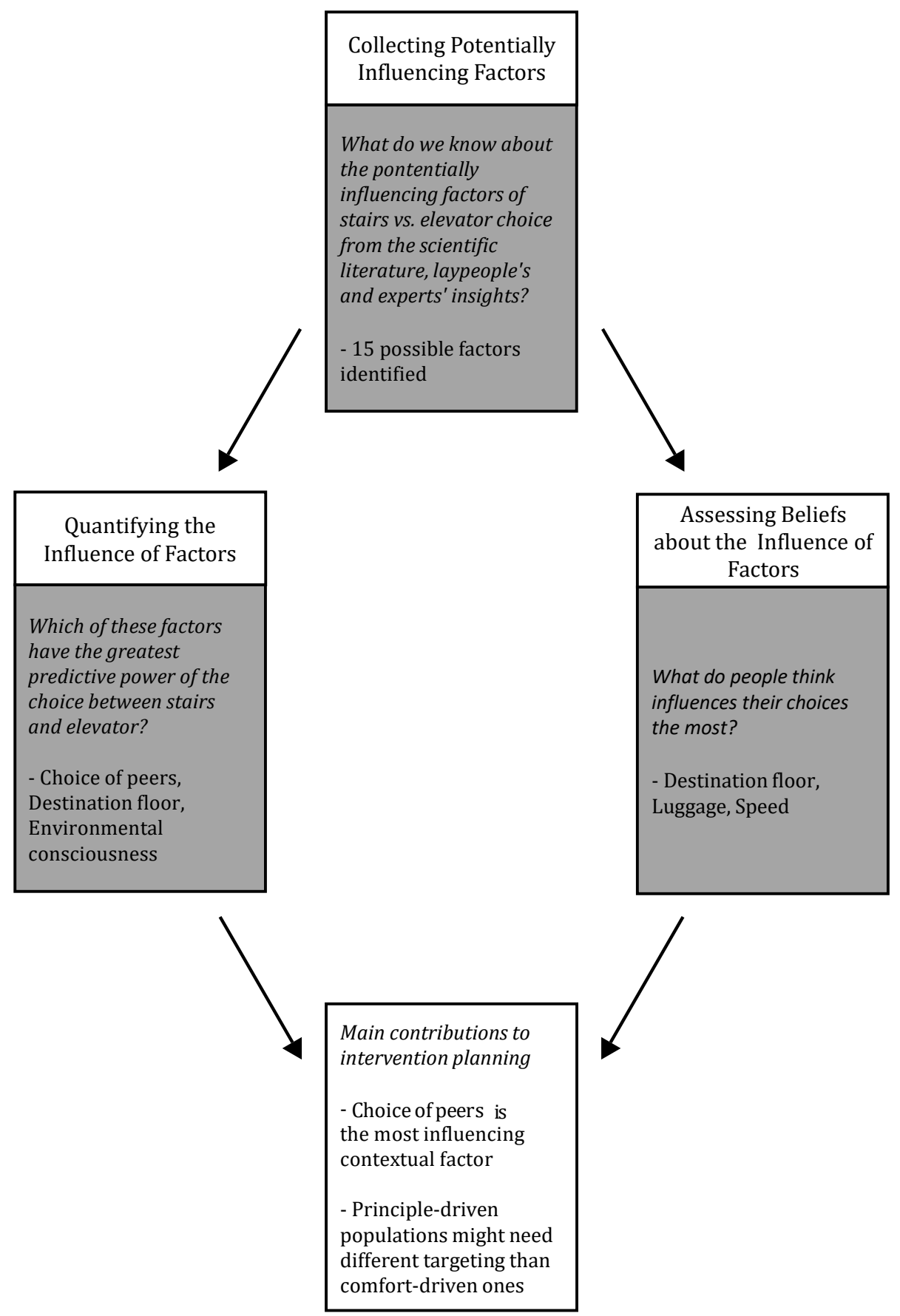

Figure 1. Steps and results of the Choice Context Mapping in the case of stairs and elevator use. 


\section{Choice Context Mapping in Practice}

Here, we demonstrate the use of Choice Context Mapping in the general choice situation when people are faced with the decision between using the stairs or elevator. It is a suitable topic of interest because multiple influencing factors might contribute to people's default choice. The variability of these unexplored factors among studies might have contributed to the mixed empirical results of this topic (Bellicha et al., 2015; Jennings et al., 2017).

Using a university student sample, we aimed to identify and assess the influencing contextual factors of stairs and elevator use as well as . Following the Choice Context Mapping method, in Step 1, we surveyed experts and laypeople in order to collect the potential factors influencing decisions between these two means of movement. In Step 2, we investigated how well the factors found in Step 1 explain individuals' behavior when choosing between taking the stairs or using the elevator. In Step 3, we study participants' beliefs about what influences their choices in this situation. Finally, we compared the results of Steps 2 and 3 to determine the degree to which the beliefs of participants correspond to their behavior.

\section{Step 1 - Collecting Potential Influencing Factors}

In Step 1, our aim was to collect a list of potential factors that might influence whether or not people use the stairs or the elevator. For this aim, we surveyed a sample from the target population (university students) as well as asked experts on the potential factors by open questions. The research plan was approved by the local institutional ethical review board.

\section{Methods}

First, we contacted 500 individuals from our local university subject pool, and successfully recruited 392 of them. They were eligible if they were at least 18 years old, and received course credits as compensation. We asked participants to list the contextual factors that they thought to influence them as well as others when choosing between stairs and elevators. Second, we collected experts by listing those who have previously published at least one peer-reviewed research article on the topic of stair usage interventions in the last decade. We asked these experts to list the potential influencing factors on stairs vs. elevator usage. Out of the 47 experts contacted, 7 
responded. (For the demographics of the respondents and the wording of the surveys, see the Supplementary Materials).

Next, each of the collected answers were processed by one member of our research team. Each time a certain type of influencing factor was mentioned, it was registered as a new category. If a newly processed answer did not belong to any of the registered categories, a new category was created. Finally, we also reviewed the relevant professional literature for additional contextual influencing factors.

Results

As a result, 15 potential influencing factors were identified: Appeal of stairs/elevator, Comfort/Laziness, Destination Floor, Elevator availability, Stairs/elevator physical availability, Environmental consciousness, Fear of confined spaces and/or technical problems, Fatigue, Importance of Health/Sports, Luggage, Number of people in the elevator, Peer behavior, Physical limitations, Speed of elevator, Temperature.

\section{Step 2 - Quantifying the Influence of Factors}

We implemented Step 2 of the procedure by collecting behavioral and contextual data to assess the degree to which the contextual factors identified in Step 1 influence the choices between stairs and elevator.

The methods and analysis procedure for Step 2 and Step 3 were pre-registered at https://osf.io/bp265. Deviations from the original pre-registered procedure are detailed in the Supplementary materials section. Data for Step 2 were collected from the same participant pool as for Step 3 in a way that participants were randomly assigned to either take part in Step 2 followed by Step 3, or in Step 3 followed by Step 2.

\section{Methods}

Participants were 523 (346 female) Hungarian university students ( $M_{\text {age }}=21.87$ years, $S D=$ 3.26) who were over 18 years old, recruited via email advertisement and received course credits as compensation. The research plan was approved by the local institutional ethical review board. 
Participants could access our online questionnaire via a link received in an email. The questionnaire first asked them to indicate whether they had visited any of the university buildings the day before; or that they did not visit higher floors that day. They were also asked to report which building they had been in the last time, as well as whether they had chosen the elevator or the stairs for going upstairs. Next, we asked them to indicate the parameters of the contextual factors at the time of the choice. For example, for the Luggage factor, they had to indicate on a 11point Likert-type scale (extremely disagree - extremely agree) how much they agreed that they were carrying a heavy luggage at the time of the choice. One exception is Peers, which was an item with 3 options where participants indicated whether they had no peers with them at the time of their choice, or whether their peers opted to use the elevator or opted to use the stairs. Only those factors identified in Step 1 were included in the questionnaire where variability in the participants' choices was expected. As a result, Physical limitations and Claustrophobia and technical problems factors were excluded, because participants indicating such physical or mental conditions would have been prevented from making a choice between using the stairs or the elevator. The items of the questionnaire are provided in the Supplement.

Participants indicating any physical or mental condition preventing them from using the stairs or the elevator (e.g., injury, claustrophobia). This accounted for two of our previously defined factors, namely Physical limitations and Claustrophobia and technical problems. We chose not to include the data of those who reported having their options limited by physical or psychological conditions, because there would have been a really low variance in their answers.

Participants received the same questionnaire on 10 consecutive weekdays. They were informed that they would receive course credit if either they reported their behavior 10 times, or reported their behavior fewer than 10 times, but were still in the top 50\% of participants' ranking based on how many times they reported among those who missed at least one occasion.

\section{Results}

According to our pre-registered analysis plan, we analyzed responses only when the participants visited an elevated floor in a university building equipped with both a set of stairs and an elevator. The data were split into training and test sets, in an $80 \%-20 \%$ ratio, respectively, in a way that every observation belonging to the same participant was included in only one of the sets. The 
training set was used for model estimation, and the test set was used to see how well the model performs on new data.

The strength of linear relationship between the contextual factors was examined by the inspection of Pearson's correlation coefficients calculated between the factors. The results showed that the highest correlation was between Environmental consciousness and Health, $r=0.77$ (for the more detailed results, see Table 1). 
Table 1. Correlations between Contextual Factors

Correlations of contextual factors

\begin{tabular}{|c|c|c|c|c|c|c|c|c|c|c|c|}
\hline & Speed & Laziness & $\begin{array}{l}\text { Destination } \\
\quad \text { floor }\end{array}$ & Fatigue & Luggage & $\begin{array}{c}\text { Elevator } \\
\text { speed }\end{array}$ & $\begin{array}{l}\text { Environmental } \\
\text { consciousness }\end{array}$ & Temperature & Appeal & $\begin{array}{l}\text { Number } \\
\text { of people } \\
\text { waiting } \\
\text { for the } \\
\text { elevator }\end{array}$ & Health \\
\hline Speed & & 0.24 & 0.37 & 0.32 & 0.25 & 0.09 & -0.19 & 0.13 & -0.11 & 0.07 & -0.18 \\
\hline Laziness & 0.24 & & 0.25 & 0.53 & 0.29 & 0.05 & -0.18 & 0.17 & -0.04 & -0.01 & -0.18 \\
\hline Destination floor & 0.37 & 0.25 & & 0.32 & 0.18 & 0.25 & -0.15 & 0.04 & -0.22 & 0.17 & -0.10 \\
\hline Fatigue & 0.32 & 0.53 & 0.32 & & 0.35 & 0.04 & -0.01 & 0.25 & -0.00 & 0.02 & -0.01 \\
\hline Luggage & 0.25 & 0.29 & 0.18 & 0.35 & & 0.01 & 0.02 & 0.37 & 0.16 & 0.11 & 0.04 \\
\hline Elevator speed & 0.09 & 0.05 & 0.25 & 0.04 & 0.01 & & 0.07 & 0.07 & -0.12 & 0.09 & 0.08 \\
\hline Environmental & -0.19 & -0.18 & -0.15 & -0.01 & 0.02 & 0.07 & & 0.17 & 0.44 & -0.02 & 0.81 \\
\hline Temperature & 0.13 & 0.17 & 0.04 & 0.25 & 0.37 & 0.07 & 0.17 & & 0.14 & 0.05 & 0.15 \\
\hline Appeal & -0.11 & -0.04 & -0.22 & -0.00 & 0.16 & -0.12 & 0.44 & 0.14 & & -0.02 & 0.45 \\
\hline Number of people & 0.07 & -0.01 & 0.17 & 0.02 & 0.11 & 0.09 & -0.02 & 0.05 & -0.02 & & -0.05 \\
\hline Health & -0.18 & -0.18 & -0.10 & -0.01 & 0.04 & 0.08 & 0.81 & 0.15 & 0.45 & -0.05 & \\
\hline
\end{tabular}


Next, in order to see the extent to what contextual factors influenced the choices made between stairs and elevators, a mixed effect logistic regression model was defined with the choices between the stairs and elevator as the dependent variable, and the measured contextual factors as independent variables. Visited buildings and IDs were treated as random effects. Speed and Destination floor were allowed to have varying slopes between different IDs, because it was plausible that these factors would have different effects on different individuals. Lasso regularization was applied to improve the interpretability and prediction accuracy of regression models by selecting only a subset of variables instead of using all of them in the final model. The lambda parameter for the Lasso regularization was chosen based on BIC values. Temperature and Number of people waiting for the elevator added the least amount of information, so their regression coefficients were penalized by the regularization process the most and were reduced to 0.

Next, we wanted to estimate how well the model explained the variation in individuals' choices. In order to do that, we estimated the variance in choosing the stairs or elevator explained by the model by calculating the squared correlation coefficient between the predicted values and the measured values, $R^{2}=0.76$.

Table 2. Regression coefficients with Standard Errors, and Odds Ratios of Contextual Factors

$\begin{array}{lccc}\text { Variables } & b & S E & O R \\ \text { (Intercept) } & 1.47 & 0.16 & 4.33 \\ & & & \\ \text { Peers - elevator } & -2.29 & 0.26 & 0.10 \\ \text { Destination floor } & -1.36 & 0.12 & 0.26 \\ & & & \\ \text { Peers - stairs } & 1.21 & 0.30 & 3.35 \\ & & & \\ \text { Environmental consciousness } & 1.02 & 0.16 & 2.77\end{array}$




\begin{tabular}{|c|c|c|}
\hline Health & 0.99 & 0.15 \\
\hline Laziness & -0.78 & 0.12 \\
\hline Elevator speed & -0.38 & 0.11 \\
\hline Speed & -0.31 & 0.11 \\
\hline Appeal & 0.23 & 0.12 \\
\hline Luggage & -0.10 & 0.11 \\
\hline Fatigue & -0.05 & 0.13 \\
\hline Temperature & 0.00 & \\
\hline $\begin{array}{l}\text { Number of people waiting for the } \\
\text { elevator }\end{array}$ & 0.00 & \\
\hline
\end{tabular}

Note. Variables are ordered by the absolute value of $b$, from largest to smallest. ORs $>1$ indicate the increase of the odds of choosing the stairs while ORs $<1$ indicate the decrease of the odds of choosing the stairs when the given feature is increased. Here the influence of Peers is represented by two coefficients. These values indicate how the probability of choosing the stairs changed when peers opted for the elevator and the stairs compared to when there were no peers present. 


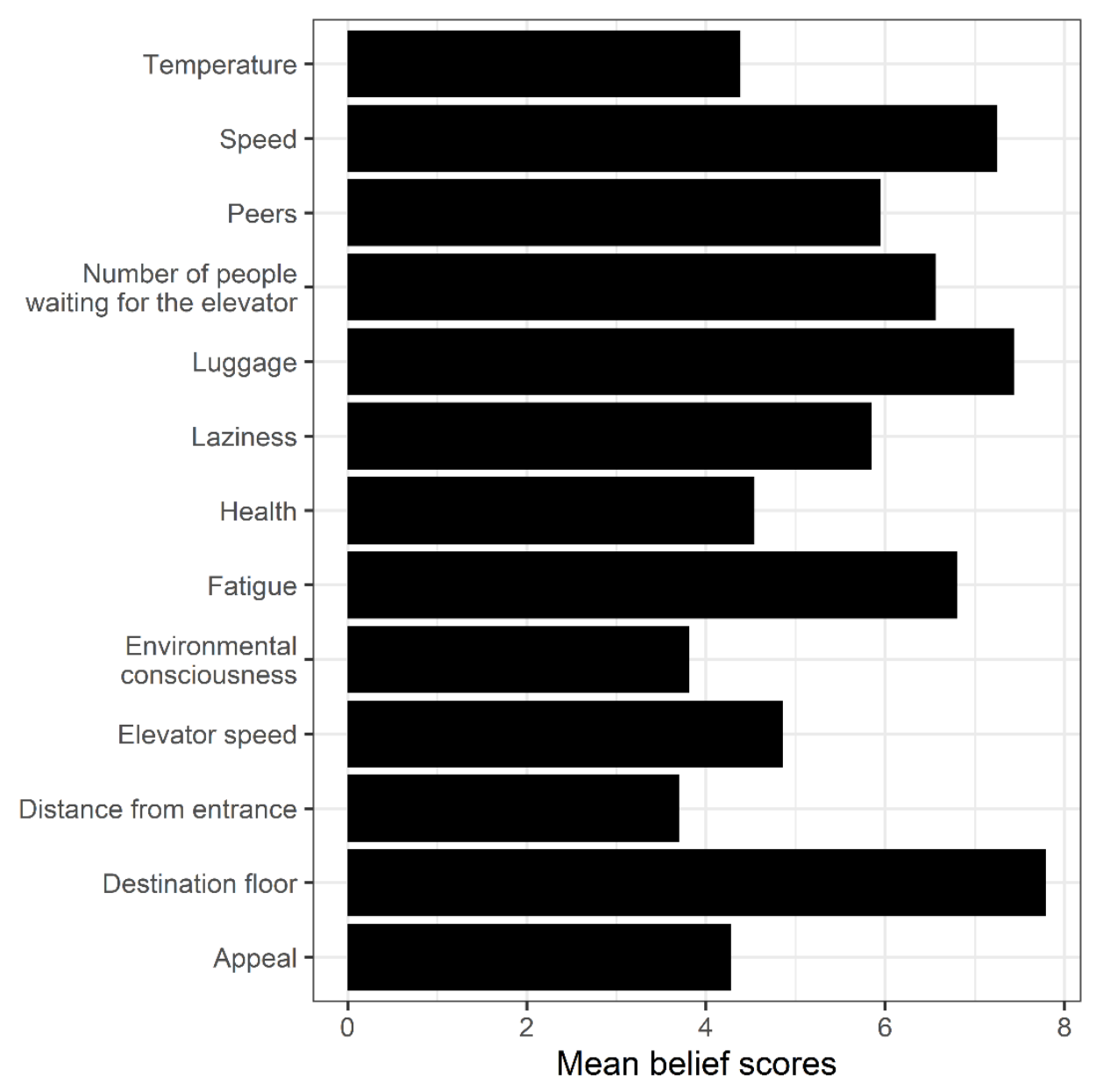

Finally, we wanted to estimate how successful our model is in correctly categorizing new data. Model predictions on the test data were compared to the real decisions to see accuracy of the model. We used a .5 probability threshold where the predicted probabilities higher than .5 were categorized as someone choosing the stairs rather than the elevator. The results showed that the model categorized $92.38 \%$ of the new cases correctly.

\section{Step 3 - Assessing Beliefs about the Influence of Factors}

During the actualization of Step 3 of the Choice Context Mapping, we aimed to measure the extent to which people believe that the collected contextual factors influence their stair/elevator choices and to explore whether people can be divided into groups that share similarities regarding their beliefs about what influences their stair/elevator choices.

\section{Methods}


We collected data from 373 (298 female, 1 did not wish to answer) university students from the same subject pool as in Step 1 and Step $2\left(M_{\text {age }}=21.86\right.$ years, $\left.S D=3.41\right)$. The research plan was approved by the local institutional ethical review board.

An online survey was created to assess beliefs regarding the believed importance of the potential contextual factors defined in Step 1, the first question being whether the participants had any physical or mental condition preventing them from using the stairs or the elevator (e.g., injury, claustrophobia). This accounted for two of our previously defined factors, namely Physical limitations and Claustrophobia and technical problems. No further questions were asked of those who reported having their options limited by physical or psychological conditions, because there would have been a really low variance in their answers. Each of the 13 factors defined in Step 2 was assessed by exactly 1 item, measuring how important the given factor was believed to be for the participants when choosing between elevators and stairs on a Likert-type scale ranging from $O$ $=$ Not important at all to $10=$ Very important . Additionally, an easy arithmetic task was placed between the questions so that unattentive answering could be detected and filtered. The survey is available at https://osf.io/xbvw8/.

Participants filled out the questionnaire either before or after the behavioral measurements of Step 2.

\section{Results}

In order to explore the individual differences regarding the factors influencing people's choices, we subjected the variables measuring the beliefs of participants to model-based clustering. This method assumes that the data come from multiple distributions, and aims to find the number of these clusters by finding their means and covariance matrices. We calculated the 10 differently parameterized models available in the mclust package in $R$ (Scrucca et al., 2016), and compared the BIC values of these models. The model with the lowest BIC value was chosen. 
Table 3. Descriptive Statistics of Beliefs

\section{Descriptive statistics - Beliefs}

\begin{tabular}{|c|c|c|c|c|c|c|c|}
\hline variable & $n$ & mean & $s d$ & median & skew & kurtosis & se \\
\hline Destination floor & 373 & 7.79 & 2.67 & 9 & -1.38 & 1.14 & 0.14 \\
\hline Luggage & 373 & 7.44 & 2.76 & 8 & -1.16 & 0.65 & 0.14 \\
\hline Speed & 373 & 7.25 & 2.79 & 8 & -1.02 & 0.29 & 0.14 \\
\hline Fatigue & 373 & 6.80 & 2.65 & 7 & -0.81 & -0.03 & 0.14 \\
\hline Number of people & 373 & 6.56 & 2.80 & 7 & -0.82 & -0.08 & 0.14 \\
\hline Peers & 373 & 5.95 & 3.02 & 7 & -0.65 & -0.61 & 0.16 \\
\hline Laziness & 373 & 5.85 & 3.01 & 6 & -0.40 & -0.86 & 0.16 \\
\hline Elevator speed & 373 & 4.86 & 2.96 & 5 & -0.15 & -1.04 & 0.15 \\
\hline Health & 373 & 4.54 & 2.96 & 5 & -0.02 & -0.97 & 0.15 \\
\hline Temperature & 373 & 4.38 & 3.27 & 4 & 0.06 & -1.35 & 0.17 \\
\hline Appeal & 373 & 4.28 & 3.03 & 5 & 0.08 & -1.08 & 0.16 \\
\hline Environmental & 373 & 3.82 & 2.95 & 3 & 0.42 & -0.82 & 0.15 \\
\hline Distance & 373 & 3.70 & 3.01 & 3 & 0.31 & -1.06 & 0.16 \\
\hline
\end{tabular}

The results show that participants can be divided into three groups in which its members hold similar beliefs about what influences their choices of stairs or elevators. Three clusters were defined and every cluster was named based on the pattern of factors. The first cluster, Efficiency group (N=118), had the highest mean scores on every scale except for Health and Environmental consciousness. Speed, Luggage, Laziness, Fatigue and Destination floor scales have the highest mean scores. The second cluster, the Health \& Environment group $(N=68)$, had their highest mean scores on the Health and Environmental consciousness scales, every other mean score was low. In the third cluster, No priority group $(N=187)$, there was no substantial difference in the group mean scores, and between the group mean scores and the sample mean scores. 
Hajdu et al. Choice Context Mapping

Figure 2. Believed importance mean scores of the potential influencing factors.

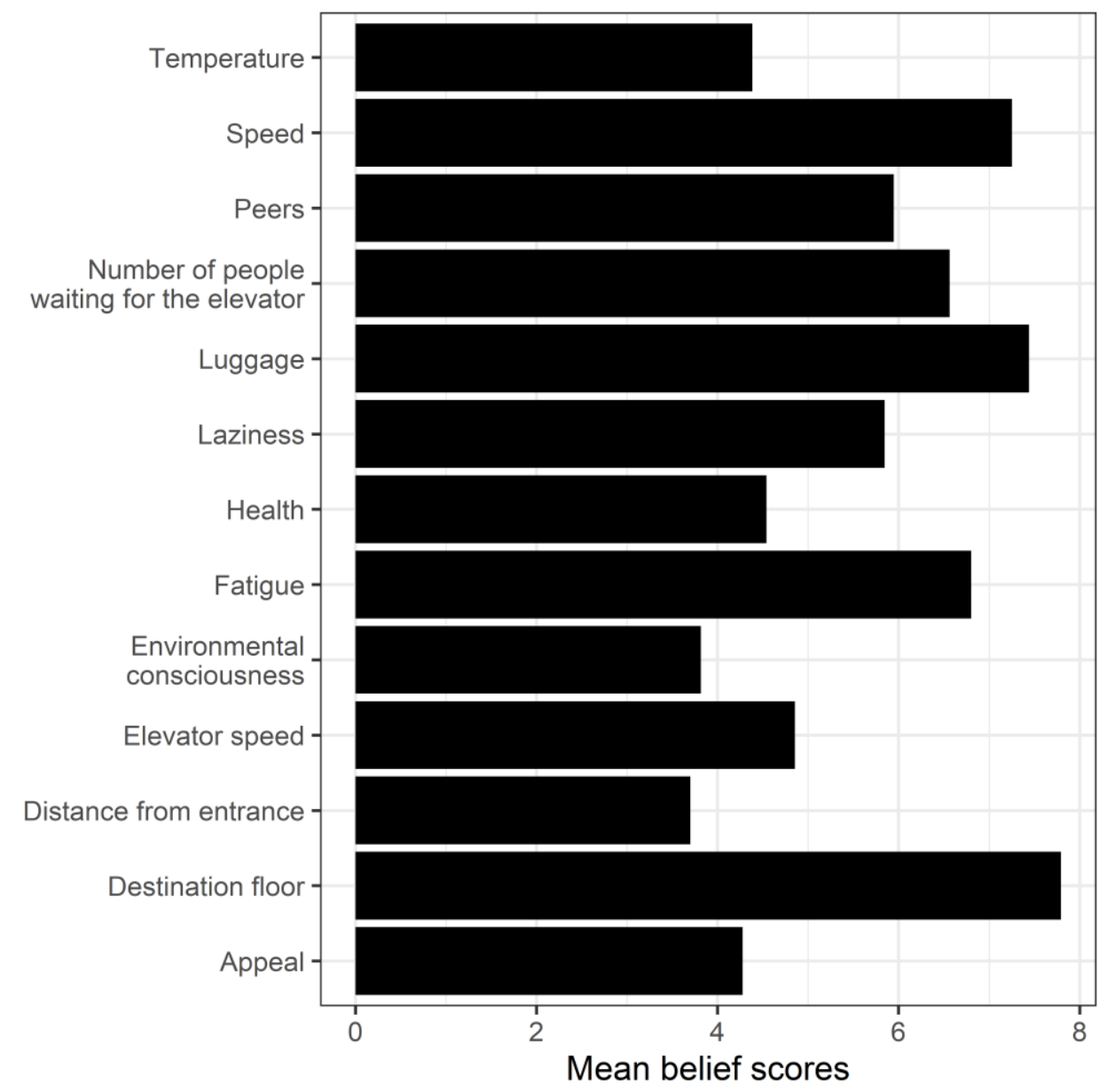


Figure 3. Believed importance standardized mean scores of the potential influencing factors by clusters.
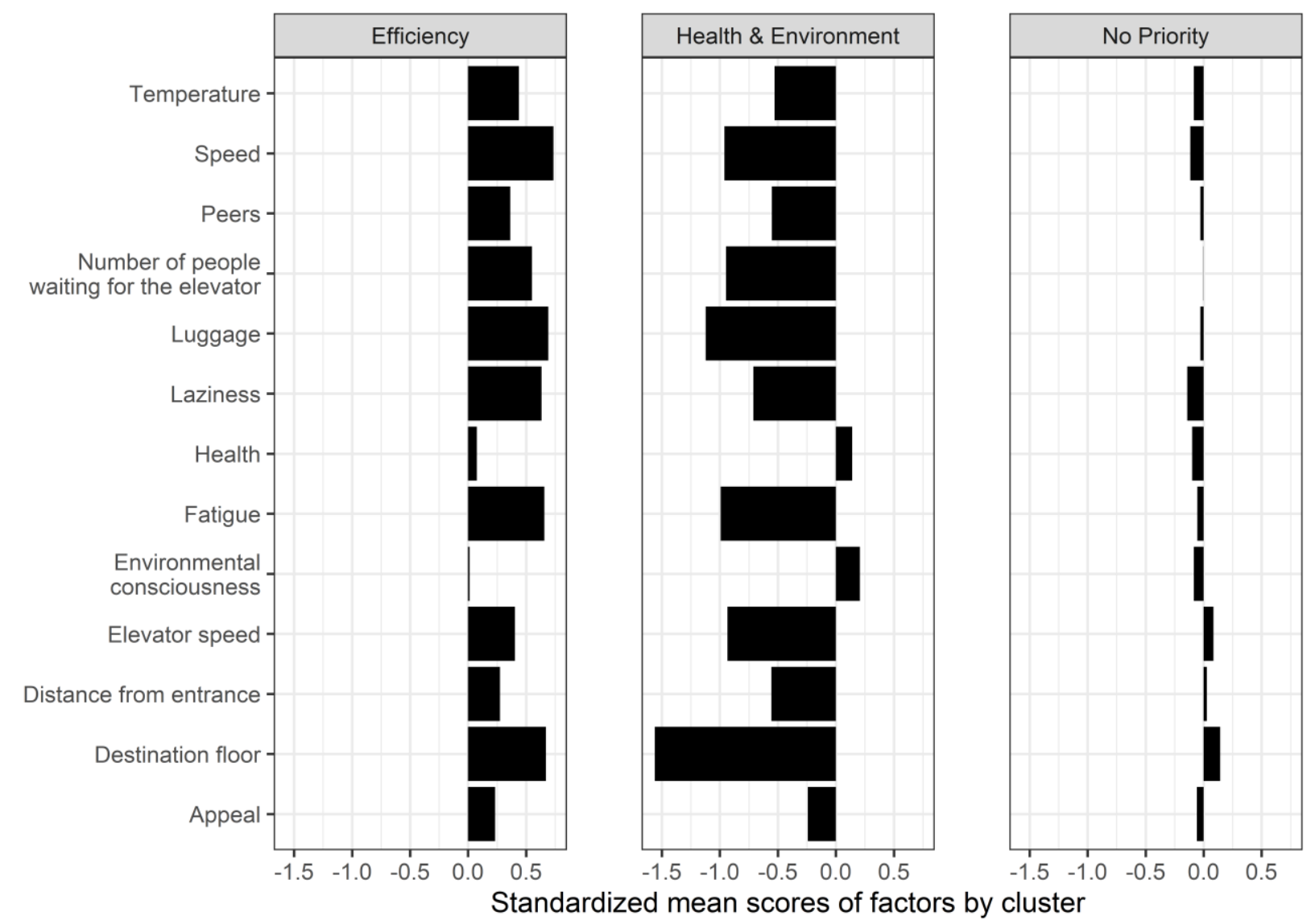

\section{Comparative analysis of Step 2 and Step 3 results}

We wished to examine whether people are right in their beliefs about what contextual factors influence them the most. To this end, we sorted the 5 factors believed to be the most influencing, based on their mean scores, as well as the 5 most influencing factors according to behavioral measurements, based on the $b$ regression coefficients from highest to lowest. Then, we compared their rankings. The first 5 beliefs with the greatest sample means are Destination floor, Luggage, Speed, Fatigue, and Number of people waiting for the elevator. From the behavioral model, the variables with the greatest $b$ coefficients are Peers ${ }^{l}$, Destination floor, Environmental consciousness, Health, and Laziness. If we take the exact rankings into consideration, there is no

\footnotetext{
${ }^{1}$ Peers is only mentioned once, the first time it appeared, because when asking participants about their beliefs, we did not make a distinction between peers influencing them to use the stairs or peers influencing them to use the elevator.
} 
match between the two sets; if we only take into account whether the given factor is in both of the sets, $20 \%$ of the factors are common.

\section{Discussion}

Practitioners of choice architecture interventions face a recurrent challenge: they have to adapt interventions to new contexts without knowing how well these interventions will perform there. This usually leads to a trial-and-error strategy, decreasing the predictability of their success. Way too often, the importance of context in the success of interventions is not realized. We argue that planning interventions should start with the thorough investigation of contextual factors of any targeted choice. This paper introduces a new procedure, the Choice Context Mapping to help intervention researchers explore the actual and believed contextual factors of situational choices. The described three steps of the procedure have been demonstrated in a concrete situation: university students' choice between elevator and stair usage.

In Step 1, we collected 15 potential contextual factors that might influence people when choosing between stairs and elevators. In Step 2, using the survey created from these factors, we estimated the effect of these factors on the participants' behavior. Based on this estimation, we managed to identify the most influential factors regarding their contribution to the studied choice. The choice of peers, the destination floor, as well as how environmentally conscious the person is, and how healthy the person aspires to be seem to have the greatest effect. The results of the analysis suggest that using the Choice Context Mapping procedure, it is possible to obtain an accurate prediction, in our case over $90 \%$, of whether one chooses the stairs or elevator based on contextual information. In Step 3, we found that participants can be divided in three discernible groups in which the members hold similar beliefs about what influences their choices of stairs or elevators. The Comfort-driven group thought their choices are mainly based on which way they think it is faster to go, whether they have any luggage, how lazy they feel, how fatigued they are and which floor they want to go to. The Principles-driven group seemed to consider which option is healthier and which is better for environmental reasons. The No priority group, which was the most numerous, believed they care equally about almost every factor. 
We have also compared people's beliefs and behavior. People seemed to assess correctly only that the destination floor is important in their choice; however, they held false beliefs about the other influencing factors. This lack of correctly evaluated factors implies that people are not really aware of what matters to them the most when they decide between taking the stairs or the elevator.

How much did we gain from applying the Choice Context Mapping in this situation? Without the exploration of our choice context, we could have missed some of these potentially influencing factors and we have had no basis of knowing the strength of these factors. This would have left us without help in knowing which factors (not) to target with our intervention. Also, without our exploration, should we have built on people's (apparently false) beliefs, we could have got misled about what contextual factors matter in their choice.

After using the Choice Context Mapping to gather relevant information, planning an intervention for this choice situation would be much easier: we already know the main factors that contribute to the choices made, the beliefs of the target population and the possible discrepancies between the two. Based on the behavioral measurements, the factors that have the greatest effect on the target behavior could be found. In our case, this was the behavior of peers. It might be worth planning future interventions around this contextual factor, such as using stimuli that accentuates the importance of the decisions of peers. Understanding the beliefs of the target population could be directly applicable for intervention planning. Based on our knowledge about the belief groups in our population, we might want to tailor our interventions that target one group more than the other; for example, Principle-driven people could be influenced more by interventions building on their identities while to influence the choices of Comfort-driven individuals one might need more expensive interventions changing the environment itself.

Choice Context Mapping can be useful in situations where choice architects face a new target choice, a new environment or a new population. The procedure can be particularly beneficial when the prevalent 'trial and error strategy' of intervention selection would be too expensive or timeconsuming. Mapping contextual influences in advance can reduce the expenses to find a working intervention, because with a better understanding of the default choice situation, the set of implementable working interventions considerably decreases. It is also a beneficial option when the risk of failed and counter-productive interventions needs to be minimized to prevent the 
negative consequences. In most cases, even if we could spare the resources and time to test every intervention first, subjecting the target population to different interventions repeatedly might diminish the effectiveness of these interventions and identifying the cause of an existing effect might be difficult.

The Choice Context Mapping has several limitations. It was designed to give a general overview of contextual factors in a choice situation, but new influencing factors could arise that previously were unaccounted for, and identified factors could change their effect over time. The dynamical changes of the influential factors could be studied with longitudinal research designs. Though Choice Context Mapping focuses on finding influential factors, their influence might come about through unexplored interactions. Models defined in Choice Context Mapping should always be tailored to be able to describe the relationships of factors; in some situations, this might be achieved with the inclusion of hierarchical models or Structural Equation Modeling, among many others.

Our results indicate that future interventions could benefit from Choice Context Mapping as a step in the planning stage. Our study focused on introducing a method for the investigation of contextual influences in a choice situation through a specific example: stairs and elevator use. We demonstrated that by going through a distinct, stringent procedure, it is possible to find the strongest contextual influencing factors of choosing the stairs or the elevator, and based on these factors, predict choices remarkably accurately. In spite of its limitations, the presented procedure is shown to be promising in terms of increasing our understanding of choice situations, and helping us in planning better interventions. Next steps regarding the exploration of the effects of moderating factors should include using Choice Context Mapping in different environments, as well as exploring these moderating factors while nudge interventions are applied. 
References

Algate, F., Gallagher, R., Nguyen, S., Ruda, S., \& Sanders, M. (n.d.). EAST.

Bellicha, A., Kieusseian, A., Fontvieille, A.-M., Tataranni, A., Charreire, H., \& Oppert, J.-M. (2015). Stair-use interventions in worksites and public settings-A systematic review of effectiveness and external validity. Preventive Medicine, 70, 3-13.

Brandon, A., Ferraro, P., List, J., Metcalfe, R., Price, M., \& Rundhammer, F. (2017). Do The Effects of Social Nudges Persist? Theory and Evidence from 38 Natural Field Experiments (No. w23277; p. w23277). National Bureau of Economic Research. https://doi.org/10.3386/w23277

Dolan, P., Hallsworth, M., Halpern, D., King, D., \& Vlaev, I. (2010). MINDSPACE: influencing behaviour for public policy.

Jennings, C. A., Yun, L., Loitz, C. C., Lee, E.-Y., \& Mummery, W. K. (2017). A systematic review of interventions to increase stair use. American Journal of Preventive Medicine, 52(1), 106-114.

John, P., Cotterill, S., Richardson, L., Moseley, A., Smith, G., Stoker, G., Wales, C., Liu, H., \& Nomura, H. (2013). Nudge, nudge, think, think: Experimenting with ways to change civic behaviour. A\&C Black.

Ly, K., Mažar, N., Zhao, M., \& Soman, D. (2013). Nudging.

Puchta, C., \& Potter, J. (2004). Focus group practice. Sage.

Schmidt, K., Schuldt-Jensen, J., Aarestrup, S. C., Jensen, A. R., Skov, K. L., \& Hansen, P. G. (2016). NUDGING SMOKE IN AIRPORTS. 8.

Scrucca, L., Fop, M., Murphy, T. B., \& Raftery, A. E. (2016). mclust 5: Clustering, classification and density estimation using Gaussian finite mixture models. The R Journal, 8(1), 289317.

Silva, A., \& John, P. (2017). Social norms don't always work: An experiment to encourage more 
efficient fees collection for students. PLOS ONE, 12(5), e0177354.

https://doi.org/10.1371/journal.pone.0177354

Szaszi, B., Palinkas, A., Palfi, B., Szollosi, A., \& Aczel, B. (2018). A Systematic Scoping Review of the Choice Architecture Movement: Toward Understanding When and Why Nudges Work: Systematic Scoping Review of the Nudge Movement. Journal of Behavioral Decision Making, 31(3), 355-366. https://doi.org/10.1002/bdm.2035

Thaler, R. H., \& Sunstein, C. R. (2008). Nudge: Improving decisions about health, wealth, and happiness. Yale University Press.

Tipton, E., Yeager, D. S., lachan, R., \& Schneider, B. (2019). Designing probability samples to study treatment effect heterogeneity. Experimental Methods in Survey Research: Techniques That Combine Random Sampling with Random Assignment, 435-456. 